\title{
The Buteyko Technique: Fake News or No News?
}

\author{
Jean-François Masse, DMD, MSc, Diplomate, ABDSM, Editor-in-Chief Journal of Dental Sleep Medicine
}

Universite Laval, Quebec City, Quebec, Canada

Innovation is what Dental Sleep Medicine is all about. Our field must be open to new ideas as it would not evolve otherwise. At a certain point however, any new idea has to be validated to enable the science to move ahead. And it is the duty of the adopters to develop well-designed studies to document what they stand for. Doing otherwise would call into question the validity and even the seriousness of the new idea that is proposed.

A trend in the management of OSA that is gaining traction bears further scrutiny: the Buteyko Breathing Method. Some advocates of the technique believe that it can help and even prevent the occurrence of obstructive sleep apnea (OSA). The technique consists of promoting nasal breathing during sleep through the use of mouth taping.

The technique has been around for quite a number of years for the treatment of asthma. However, very few rigorous studies have examined the impact of the Buteyko method on asthma. A 2005 review underlined the lack of research on the topic, and a 2009 randomized single-blind crossover study found the technique had no effect on asthma control. ${ }^{1,2}$ The small number of carefully controlled studies suggests that the medical community has seen little value in the Buteyko method.

When examining the OSA scientific literature, one realizes there are no established protocols to treat sleep patients using the Buteyko technique. In fact, the only article on Buteyko and sleep apnea available on Pubmed is a 2012 survey published by the Australian Nursing Journal. ${ }^{3}$ Saying that the evidence is thin here is an understatement...

Does this mean that the Buteyko technique is worthless? We simply do not know as there is so little evidence available. Those who believe in the method and promote it need to engage in quality research if they want to be taken seriously. I suggest they first take a close look at a very interesting pilot study published in the JDSM of January 2018 by Milano et al. ${ }^{4}$ The study suggests that vertical elastics that minimize mouth opening may enhance the outcome of oral appliance treatment. When the mouth is closed, nasal breathing is obligatory, however the relationship between tongue position, mandibular position and nasal breathing with regard to obstructive sleep apnea has yet to be evaluated.
Until further studies are conducted, caution should be exercised when promoting the Buteyko method or any unvalidated technique. Our community has worked hard to develop a field of practice based on sound scientific principles, and we cannot afford at this point to endorse treatment methods based only on anecdotal evidence.

\section{CITATION}

Masse JF. The Buteyko technique: fake news or no news. Journal of Dental Sleep Medicine. 2018;5(3):53-54.

\section{REFERENCES}

1. Bruton A, Lewith GT. The Buteyko breathing technique for asthma: a review. Complement Ther Med. 2005;13(1):41-6.

2. Cooper S, Oborne J, Harrison T, Tattersfield A. Effect of mouth taping at night on asthma control--a randomised single-blind crossover study. Respir Med. 2009;103(6):813-9. doi: 10.1016/j.rmed.2009.02.003.

3. Birch M . Sleep apnoea: a survey of breathing retraining. Aust Nurs J. 2012;20(4):40-1.

4. Milano F, Mutinelli S, Sutherland K, Milioli G, Scaramuzzino G, Bacchieri Cortesi A, Siciliani G, Lombardo L, Cistulli P. Influence of Vertical Mouth Opening on Oral Appliance Treatment Outcome in Positional Obstructive Sleep Apnea. JDSM. 2018;5(2):17-23 


\section{SUBMISSION \& CORRESPONDENCE INFORMATION}

Submitted for publication June 26, 2018

Submitted in final revised form June 27, 2018

Accepted for publication June 27, 2018

Address correspondence to: Jean-François Masse, Professor, Universite Laval, 2780 Masson \#200, Quebec City, QC, G1P 1J6, Canada; Tel: 418- 871-1447; Fax: 418-871-4983; Email: jeanfrancois.masse@fmd.ulaval.ca 